\title{
Multi-Dimensional Integration and Packaging of Devices for Internet-of-Things Applications
}

\author{
Nazek El-Atab \\ mmh Labs, Computer, Elecctrical \\ and Mathematical Sciences and \\ Engineering Division \\ King Abdullah University of \\ Science and Technology \\ Thuwal, Saudi Arabia \\ nazek.elatab@kaust.edu.sa
}

\begin{tabular}{c} 
Reema Almansour \\
Electrical Engineering \\
Department, Princess Nourah \\
Bint Abdul Rahman University \\
Riyadh, Saudi Arabia \\
Reema.mansour@kaust.edu.sa \\
\hline
\end{tabular}

Reema Almansour Electrical Engineering
Department, Princess Noura Abdul Rahman University Reema.mansour@kaust.edu.sa

\author{
Reema Suwaidan \\ Electrical Engineering \\ Department, Princess Nourah \\ Bint Abdul Rahman University \\ Riyadh, Saudi Arabia \\ reema.suwaidan@kaust.edu.sa
}

Sohail F. Shaikh

mmh Labs, Computer, Elecctrical and Mathematical Sciences and Engineering Division

King Abdullah University of

Science and Technology

Thuwal, Saudi Arabia

sohailfaizan.shaikh@kaust.edu.sa

\author{
Yara Alghamdi \\ Electrical Engineering \\ Department, Princess Nourah \\ Bint Abdul Rahman University \\ Riyadh, Saudi Arabia \\ yara.ghamdi@kaust.edu.sa
}

Sherjeel Khan
mmh Labs, Computer,
Elecctrical and Mathematical
Sciences and Engineering
Division
King Abdullah University of
Science and Technology
Thuwal, Saudi Arabia
sherjeel.khan@kaust.edu.sa

\author{
Alhanouf Alhazzany \\ Electrical Engineering \\ Department, Princess Nourah \\ Bint Abdul Rahman University \\ Riyadh, Saudi Arabia \\ Alhanouf.hazzany@kaust.edu.sa
}

\begin{abstract}
IoT applications are increasingly becoming widespread with more stringent system requirements. In this work, we demonstrate a nature-inspired integration and packaging technology that achieves self-powered multi-functional systems with optimized performance and small footprint area. The integration technique is based on bifacial usage of the substrate where devices on both sides are interconnected via through-substrate-vias. Multiple substrates are then integrated and folded into a 3D architecture using side-interlocks following a puzzle-like fashion. On the outer sides of the 3D architecture, sensors, RF devices and energy harvesters are integrated while on the inner faces, a solid-state battery in addition to powermanagement and data-management circuitry are embedded. To package the system, a polymeric encapsulant is used to protect the inner circuitry and enhance the mechanical resilience of the system. Finally, the system is used to send the collected data wirelessly to a phone using an embedded Bluetooth Low Energy unit.
\end{abstract}

Keywords-integration, packaging, system-on-chip, system-inpackage, sensors.

\section{INTRODUCTION}

Since its emergence, Internet-of-Things (IoT) has brought myriads of benefits to our lives across multiple fields including healthcare, defense and transportation, where "intelligent" sensors intercommunicate seamlessly over the internet without the need for human intervention [1-16]. In this light, the promise of a smart environment has been exceptionally attractive where the physical and the artificial worlds interact

This work is supported by King Abdullah University of Science and Technology (KAUST) Office of Sponsored Research (OSR) under Award No. Sensor Innovation Initiative OSR - 2015 - Sensors - 2707 and KAUST KFUPM Special Initiative OSR - 2016 - KKI - 2880. and generate data that contributes to a smarter and more comfortable world. Nevertheless, the advances in IoT resulted in more and more stringent requirements at all levels including hardware, software and communications. More specifically, IoT devices need to be more multifunctional than ever with high performance, low power consumption and small footprint area. As a result, the prevailing integration and packaging technologies are continuously developing to meet such requirements.

The most popular integration and packaging approaches have been using system-on-chip $(\mathrm{SoC})$ and system-in-package (SiP) techniques. However, each of these technologies has its own shortcomings. For instance, the SoC technique is restricted to what can be accomplished within a single wafer process leading to an imperfect performance of multiple components while $\mathrm{SiC}$ is bulky and is restricted to the performance of CMOS components which are not always optimal for specific applications [3-31]. To bridge the gap between the two technologies, here, we propose an approach based on multi-dimensional integration and packaging of devices where both sides of a substrate are utilized for thin-film based device fabrication. Both faces of the substrate are interconnected via through-substrate-vias (TSVs). The multiple faces of the multidimensional integrated circuit (MD-IC) are interconnected using side interlocks to achieve a small form factor. Finally, a solution-processable polymer is used to encapsulate the system and improve its mechanical resilience. The MD-IC integration and packaging technique enables an optimized performance of the system since each side can be 
based on a different substrate material with heterogeneous devices. In addition, since the devices on each side can be fabricated separately, then heterogeneous devices can be integrated with no need for costly and complicated lithography masks.

\section{INTEGRATION OF DEVICES}

Inspired by nature, where human's different sensors are integrated on the body outer side such as touch, smell and sight (Fig.1), while the memory, logic and power are embedded inside the body (i.e. brain), our proposed MD-IC integrates sensors on the external sides of the $3 \mathrm{D}$ electronic architecture while the power management and data management circuitry are added on the internal sides.

\section{A. Devices Integration}

Traditionally, the process flow of integration and packaging of devices consists of the fabrication of the devices on a silicon wafer followed by dicing it into smaller dies which are then packaged using a ceramic material. Our CMOS-compatible integration and packaging technique introduces a few variations to the traditional approach to achieve MD-ICs. First, on the polished-side of a 4 inch silicon wafer, multiple devices are fabricated such as high-performance microprocessors, which is a fabrication process that require a high thermal budget. The devices are then protected using a mask and fabrication of devices on the back-side of the wafer takes place.

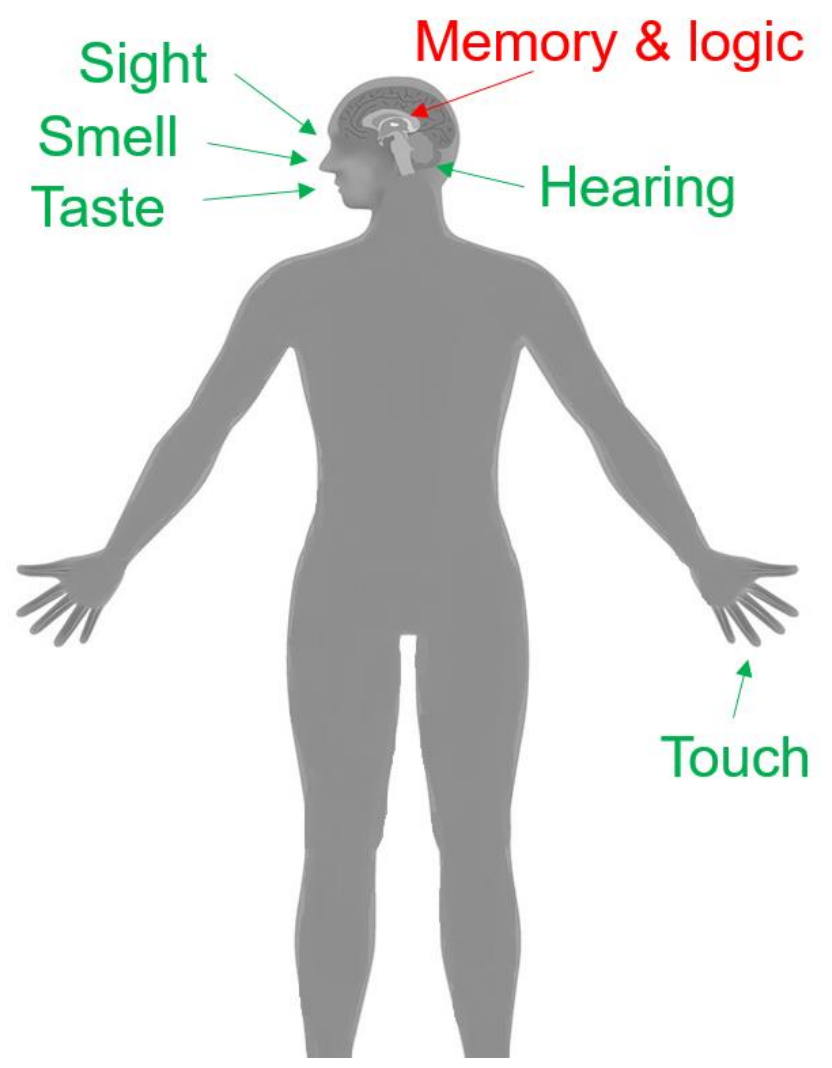

Fig. 1. Nature inspiration for the demonstrated MD-IC where sensors are integrated on the external faces while power and data management circuitry are added on the internal faces.

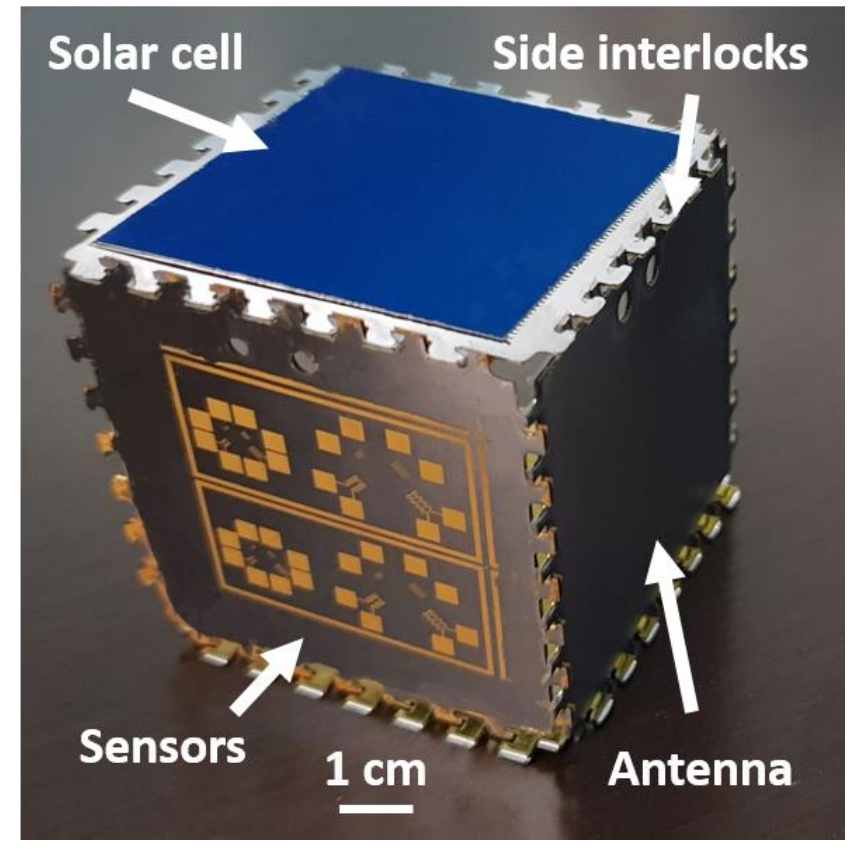

Fig. 2. Cubic MD-IC with integrated sensors, RF and energy harvesters on its outer sides.

The back-side of the wafer is unpolished and shows a higher defects density, thus, devices that do not need the substrate as an active material would be integrated such as sensors. It is also important to mention that the fabrication on the rear-side of the Si sample should require a low thermal budget to avoid deteriorating the already embedded devices on the top polished side. Next, the backside of the wafer is protected with photoresist and a kapton mask and patterned using a $\mathrm{CO}_{2}$ laser.

The exposed areas are then completely etched following the Bosch process in a deep reactive ion etching system (DRIE). This results in the various sides of the MD-IC with side interlocks and TSVs which are then coated/filled with a

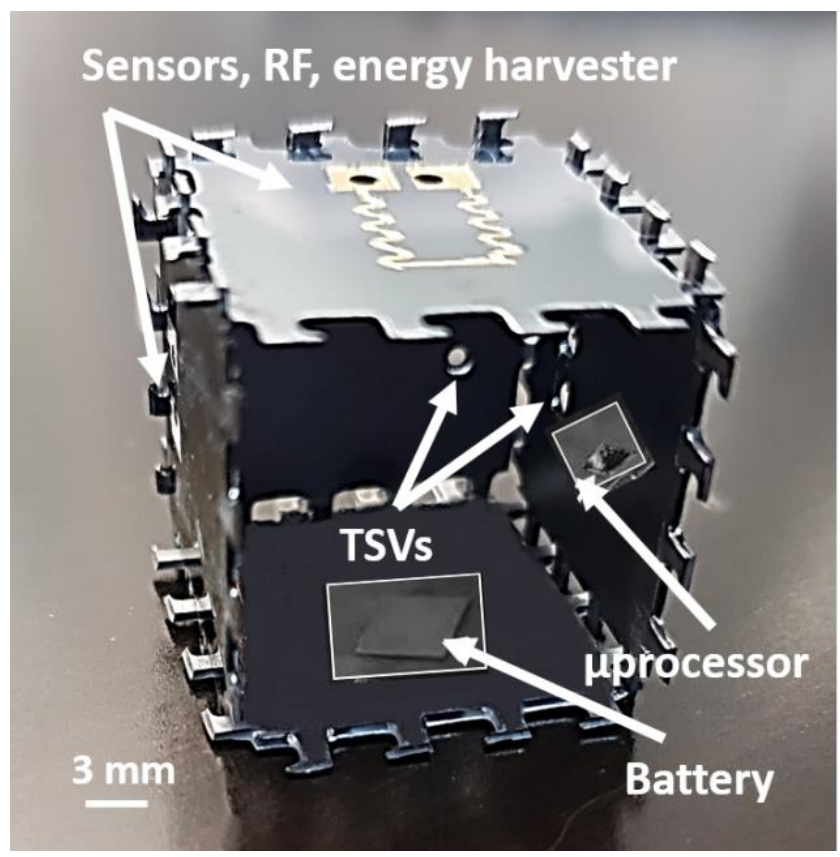

Fig. 3. Cubic MD-IC with integrated power and data management circuitry on its inner sides. 


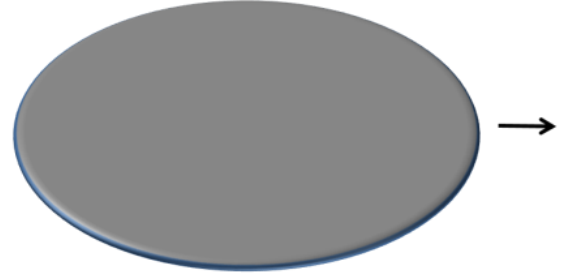

Single side polished wafer

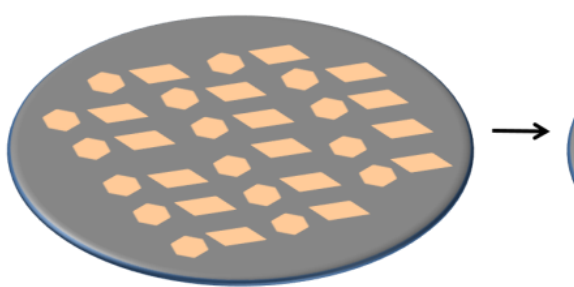

Fabrication of $\mu$ processors (polished side, high thermal budget)

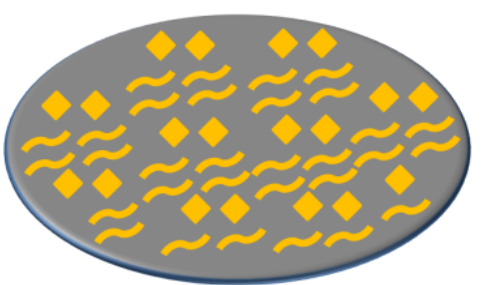

Fabrication of Antennas, sensors, TSVs (unpolished side, low thermal budget)
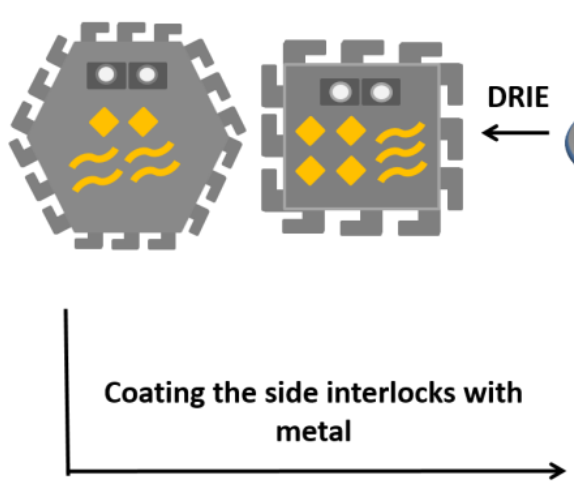
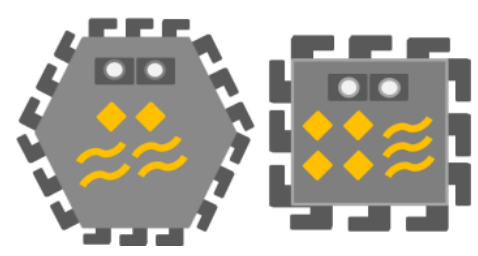

Patterning

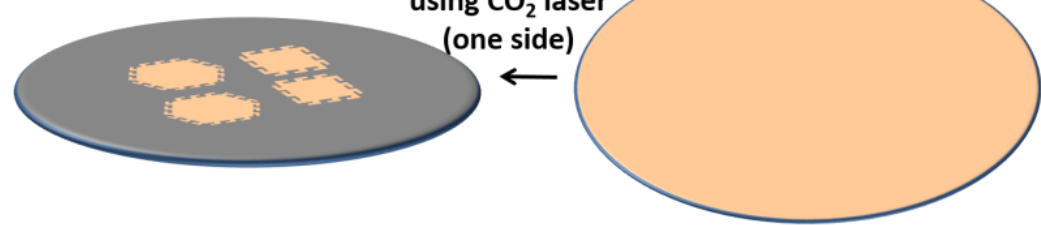

Application of Photoresist and Polymer (Kapton) on both sides

Fig. 4. CMOS compatible fabrication process flow of the MD-ICs. Devices on the outer-side and inner-side faces of the substrate are interconnected through TSVs while side to side devices are interconnected through side interlocks in a puzzle-like manner.

metallic layer. It is worth to mention that the size of the faces, the dimensionality (cube, pyramid, etc.), the number of TSVs and side interlocks can be adjusted and tailored based on the application requirements. Finally, the various faces of the MDIC are folded together into a 3D architecture in a puzzle-like fashion. Fig. 2 and Fig. 3 show a cubic MD-IC with multiple sensors and a silicon solar cell [32-33] integrated on the outer faces while power and data management circuitry are embedded on the inner faces, respectively. The thorough fabrication process flow is shown in Fig. 4. It is important to mention that each side of the MD-IC can use a different material in an attempt to boost the performance of the full system.

\section{B. Devices Characterization}

As a proof of concept, multiple sensors ae fabricated and integrated in the MD-IC including $\mathrm{pH}$, pressure and ammonia level sensors. The $\mathrm{pH}$ sensor is resistive based and uses a graphene monolayer as the active material. The sensor is developed by first depositing an $\mathrm{SiO}_{2}$ insulating layer using plasma enhanced chemical vapor deposition system (PECVD) on a Si wafer. Next, interdigitated contacts are deposited using a sputtering system and patterned using a reactive ion etching tool (RIE). Finally, a graphene monolayer is transferred onto the interdigitated contacts and left to dry at room temperature. The sensor is then tested using solutions with different $\mathrm{pH}$ values. The resistance of the sensor is shown to reduce at larger
$\mathrm{pH}$ values. In fact, the $\mathrm{OH}^{-}$and $\mathrm{H}_{3} \mathrm{O}^{+}$result in a doping effect in graphene which affects its conductivity. $\mathrm{OH}^{-}$ions are shown to cause a greater conductivity in the graphene layer than $\mathrm{H}_{3} \mathrm{O}^{+}$ ions (Fig. 5).

The pressure sensor uses the same structure as the $\mathrm{pH}$ sensor with a graphene monolayer as the active material. In this

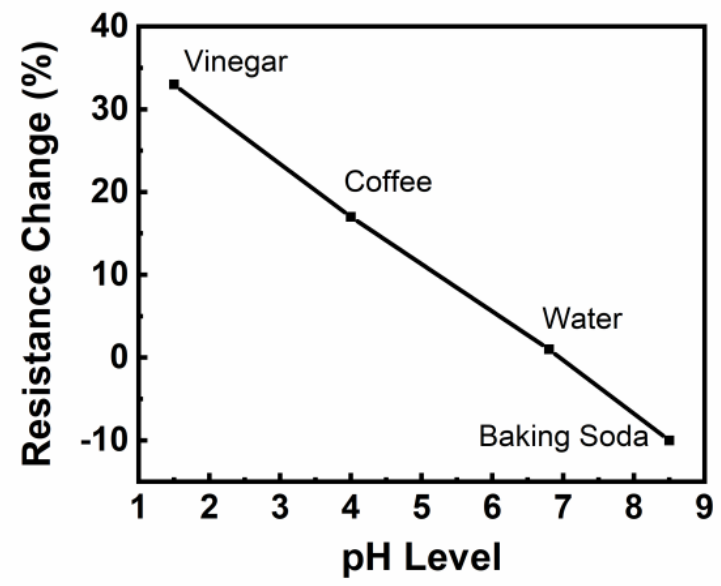

Fig. 5. $\mathrm{pH}$ sensor response when exposed to solutions with different $\mathrm{pH}$ values. 


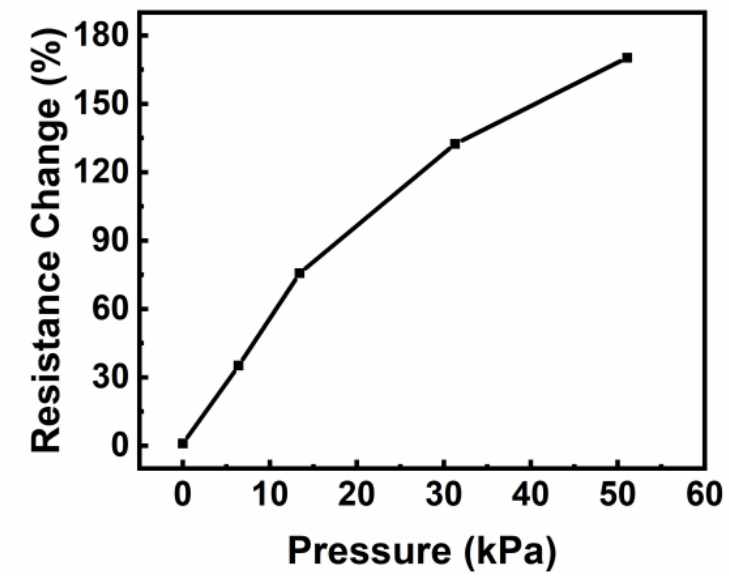

Fig. 6. Pressure sensor response when exposed to different weights.

case, the pressure sensor shows an increased resistance with pressure (Fig. 6), in fact, it has been reported that as the applied pressure on the graphene increases, the electron density increases while the electron mobility decreases. However, the electron mobility degradation over-compensates the electron density enhancement which results in an overall increase in resistance [34-42].

Finally, the ammonia level sensor uses laser induced graphene where a polyimide sheet was laser ablated using a $\mathrm{CO}_{2}$ laser. The obtained graphene layer is around and shows defects. Such defects are favored because they increase the sensitivity of the ammonia level sensor. Different structures are

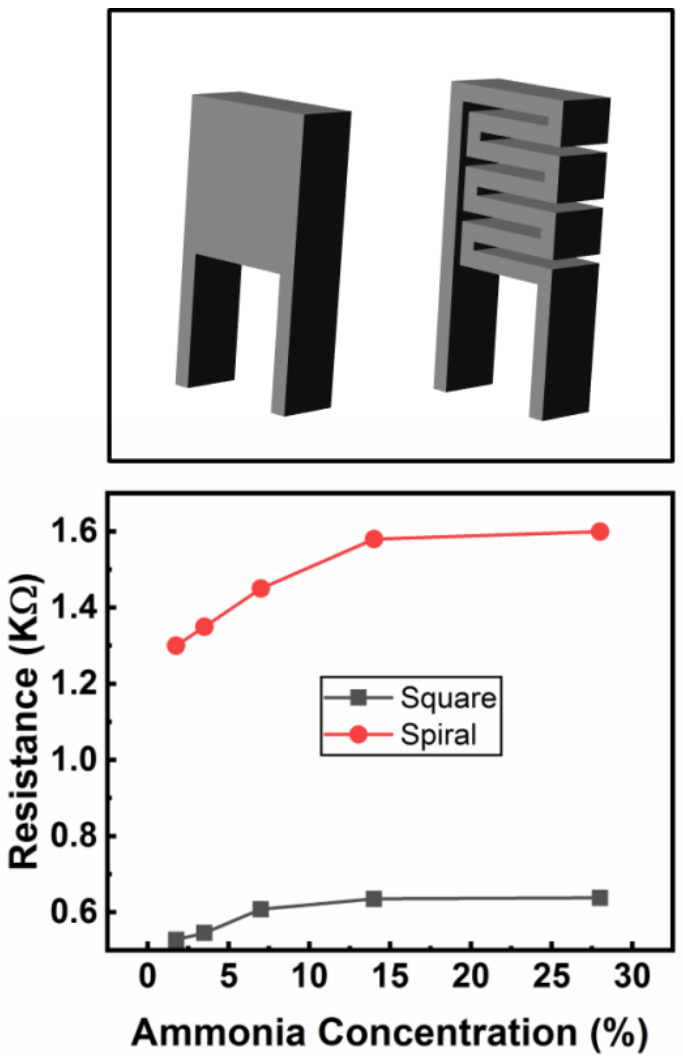

Fig. 7. Ammonia level sensor response when exposed to ammonia concentrations where two distinct sensor structures are studied. studied in this case, a square structure vs. a spiral structure with a higher surface area-to-volume ratio. Consequently, the sensitivity of the sensor in improved using the spiral structure as shown in Fig. 7.

\section{PACKAGING}

Once folded into the 3D architecture (cubic architecture in the demonstrated prototype), the MD-IC is encapsulated using a solution processable polymer: polydimethylsiloxane (PDMS). The PDMS is prepared by mixing 10:1 ratio of silicone elastomer: curing agent. The solution is then further mixed under vacuum for $4 \mathrm{~min}$ to remove any air bubbles. Finally, the obtained PDMS is poured on each face of the cubic MD-IC and cured at $60^{\circ} \mathrm{C}$ for 2 hours. Next, $\mathrm{CO}_{2}$ laser is used to pattern the PDMS and expose the sensors that necessitate direct contact with the external environment as shown in Fig. 8 . The used laser power is $24 \mathrm{~W}$ with a speed of $2 \mathrm{~mm} / \mathrm{s}$ and a height of $1 \mathrm{~mm}$.

It is worth to mention that the faces that do not require exposure to the outer environment can remain fully encapsulated. PDMS has a very low thermal conductivity and thus contributes to reducing the heat transfer from the outside to the inner devices [43]. This characteristic would be helpful especially for applications of the MD-IC in harsh environments. To test this, a single side of the MD-IC was imaged under an infrared microscope when the chuck is heated up to $80^{\circ} \mathrm{C}$. The encapsulated side showed around $20^{\circ} \mathrm{C}$ difference compared to the un-encapsulated side as shown in the thermal image depicted in Fig. 9.

Moreover, the PDMS enhances the mechanical robustness of the complete system. In fact, the encapsulated MD-IC was dropped from different heights (up to $15 \mathrm{~m}$ ) using a drone tool empowered with a 3D printed object-dropping system where the PDMS was able to completely absorb the mechanical

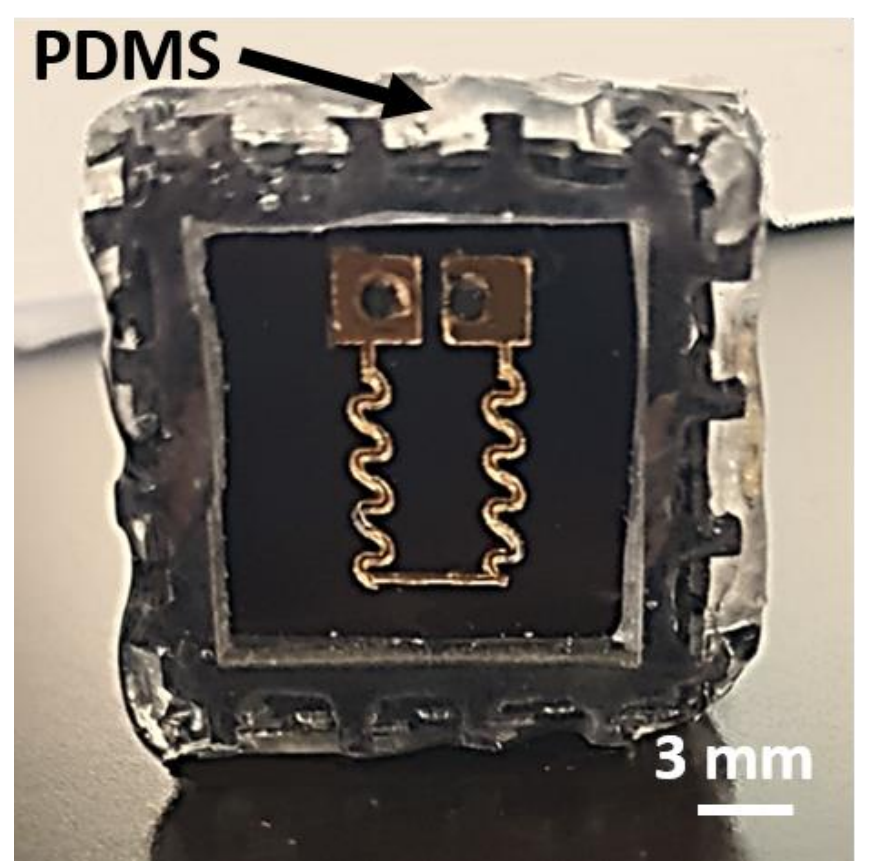

Fig. 8. Encapsulated side of the MD-IC using PDMS. $\mathrm{CO}_{2}$ laser is used to expose the sensor to the outer environment. 


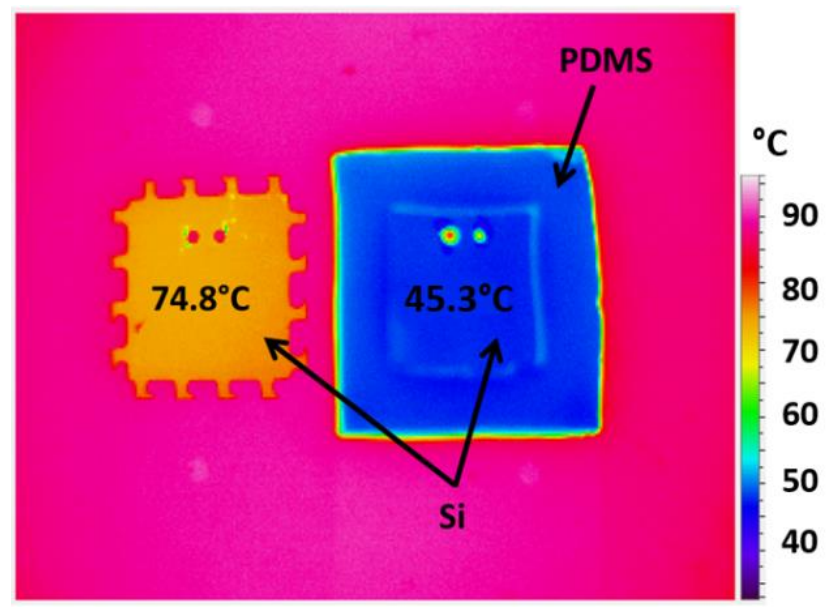

Fig. 9. Thermal image of the MD-IC side (encapsulated vs bare) when the chuck is heated up to $80^{\circ} \mathrm{C}$.

shocks, while un-encapsulated silicon would completely crack when dropped from heights below $2 \mathrm{~m}$.

Finally, a system reliability test is conducted on the MD-IC where multiple devices are embedded on the different sides and different faces such that they are interconnected through the TSVs and side interlocks. The devices include a temperature sensor, a solid-state battery and a microcontroller with a Bluetooth Low Energy chip (BLE). As a proof-of-concept, we show the data being read and processed by the microcontroller at and the result is sent by Bluetooth to a phone. A basic and free mobile application $\left(\mathrm{CySmart}^{\mathrm{TM}}\right)$ is used to show the sent data by Bluethooth as a proof-of-concept. However, it is worth to note that more advanced applications can be developed to show multiple collected datasets on the same screen. In addition, more memory devices can be integrated to make the IoT system smarter [44-46]. In this reliability test, the encapsulated MD-IC with PDMS is placed in a beaker filled with water. The beaker is then placed on a hot plate, as a result, the detected temperature sent to the phone is shown to rise with time. The beaker is then placed on a cold surface where the temperature is shown to reduce again. This test verifies the potential of the MD-IC in integrating heterogeneous device on its different faces and sides.

\section{CONCLUSION}

In this work, we demonstrated a new design concept which is nature-inspired and specifically focused on standalone IoT systems. The multi-dimensional integration and packaging of devices bridges the gap between the most successfully existing integration and packaging technologies (SoC and $\mathrm{SiP}$ ) and thus, it enables heterogeneous integration of materials and devices on different sides and faces of the system. The different sides are then folded into a 3D architecture in a puzzle-like fashion. The devices are interconnected through TSVs and side interlocks. The MD-IC allows for a high performance system for IoT applications with a small footprint area. In addition, since the heterogeneous devices can be fabricated on the different sides separately, then additional complicated lithography masks are not needed which reduces its cost. Finally, as a proof of concept, we show the application of the MD-IC in collecting the temperature of water and sending it to a phone using the Bluetooth technology.

\section{ACKNOWLEDGMENT}

M.M.H. conceptualized the idea and directed the study. N.E.A. directed the study, developed the integration and packaging technology, characterized the fabricated devices, and assessed and analyzed the findings. A.A. and R.A. assisted in the development of the ammonium hydroxide sensors. Y.A. and R.S. helped in the development of the salinity sensor. S.F.S and S.M.K helped in the characterization of the microcontroller.

\section{REFERENCES}

[1] N. El-Atab, S. Shaikh, S. Khan and M. Hussain, "Bi-Facial Substrates Enabled Heterogeneous Multi-Dimensional Integrated Circuits (MD-IC) for Internet of Things (IoT) Applications", Advanced Engineering Materials, vol. 21, no. 7, p. 1900043, 2019. Available: 10.1002/adem.201900043.

[2] N. El-Atab et al., "Heterogeneous Cubic Multidimensional Integrated Circuit for Water and Food Security in Fish Farming Ponds", Small, p. 1905399, 2019. Available: 10.1002/smll.201905399.

[3] R. Esfandyarpour, M. J. DiDonato, Y. Yang, N. Gozde Durmus, J. S. Harris, and R. W. Davis "Multifunctional, inexpensive, and reusable nanoparticle-printed biochip for cell manipulation and diagnosis." Proceedings of the National Academy of Sciences, 201621318, 2017.

[4] H. Mohammad Fahad, H. Shiraki, M.Amani, C. Zhang, V. Srinivas Hebbar, W. Gao, H. Ota, M. Hettick, D. Kiriya, Y.-Z Chen, Y.L. Chueh and A. Javey. "Room temperature multiplexed gas sensing using chemical-sensitive 3.5-nm-thin silicon transistors." Science advances 3, no. 3, 2017.

[5] M. Mimee, P. Nadeau, A. Hayward, S. Carim, S. Flanagan, L. Jerger, J. Collins et al.. "An ingestible bacterial-electronic system to monitor gastrointestinal health.” Science, 360(6391), 915-918, 2018.

[6] D. J. Poxson, M. Karady, R. Gabrielsson, A. Y. Alkattan, A. Gustavsson, S. M. Doyle, S. Robert et al. "Regulating plant physiology with organic electronics." Proceedings of the National Academy of Sciences, 114(18), 4597-4602, 2017.

[7] Y. Lee, C. Howe, S. Mishra, D. Sup Lee, M. Mahmood, M. Piper, Y. $\mathrm{Kim}$ et al. "Wireless, intraoral hybrid electronics for real-time quantification of sodium intake toward hypertension management." Proceedings of the National Academy of Sciences, 115(21), 5377-5382, 2018.

[8] M. Bariya, H. Yin Yin Nyein, and A. Javey. "Wearable sweat sensors." Nature Electronics, 1(3), p.160, 2018.

[9] C. Jungil, R. Ghaffari, L. B. Baker, and J. A. Rogers. "Skin-interfaced systems for sweat collection and analytics." Science Advances 4, no. 2, 3921, 2018.

[10] S. M. Khan, A. Gumus, J. M. Nassar, and M. M. Hussain "Personalized Healthcare: CMOS Enabled Microfluidic Systems for Healthcare Based Applications." Advanced Materials, 30(16), p.1870111, 2018

[11] D. Seo, R. M. Neely, K. Shen, U. Singhal, E. Alon, J. M. Rabaey, J. M. Carmena, and M. M. Maharbiz. "Wireless recording in the peripheral nervous system with ultrasonic neural dust." Neuron vol. 91, pp. 529-539, 2016

[12] M. Alioto, E. Sánchez-Sinencio, \& A. Sangiovanni-Vincentelli. "Guest Editorial Special Issue on Circuits and Systems for the Internet of Things-From Sensing to Sensemaking." IEEE Transactions on Circuits and Systems I: Regular Papers, 64(9), 2221-2225, 2017.

[13] S. Sathyan, \& S. R. A Pulari. "Deeper Insight on Developments and RealTime Applications of Smart Dust Particle Sensor Technology." In Computational Vision and Bio Inspired Computing (pp. 193-204). Springer, Cham, 2018.

[14] J.A. Rogers, T. Someya, \& Y. Huang. "Materials and mechanics for stretchable electronics." Science, 327(5973), 1603-1607, 2010. 
[15] K. Takei, Z. Yu, M. Zheng, H. Ota, T. Takahashi, A. Javey. "Highly sensitive electronic whiskers based on patterned carbon nanotube and silver nanoparticle composite films." Proceedings of the National Academy of Sciences, 111(5), 1703-1707, 2014.

[16] C.H. Lee, D.R. Kim, \& X. Zheng. "Fabricating nanowire devices on diverse substrates by simple transfer-printing methods." Proceedings of the National Academy of Sciences, 2010

[17] R. Aitken, V. Chandra, J. Myers, B. Sandhu, L. Shifren, and G. Yeric. "Device and technology implications of the internet of things" " In 2014 Symposium on VLSI Technology (VLSI-Technology): Digest of Technical Papers, pp. 1-4, 2014.

[18] L. Davoli, L. Belli, A. Cilfone, and G.i Ferrari. "From micro to macro IoT: Challenges and solutions in the integration of IEEE 802.15. 4/802.11 and Sub-GHz technologies." IEEE Internet of Things Journal 5, no. 2, 784-793, 2018

[19] A. Tai-Ying, J. Lee, D. Lee, and C.-Chih Chen. "The development of IC packaging under the Internet of Things standards." In 2016 11th International Microsystems, Packaging, Assembly and Circuits Technology Conference (IMPACT), pp. 209-211, 2016.

[20] S. Pendharkar. "Smart power technologies enabling power SoC and SiP." In 2016 IEEE Symposium on VLSI Technology, pp. 1-2, 2016.

[21] G. Carchon, X. Sun, G. Posada, D. Linten, and E. Beyne. "Thin-film as enabling passive integration technology for RF SoC and SiP." In 2005 IEEE International Solid-State Circuits Conference, 2005. Digest of Technical Papers. ISSCC. pp. 398-399, 2005.

[22] K. L. Tai. "System-In-Package (SIP): challenges and opportunities." In Design Automation Conference, 2000. Proceedings of the ASP-DAC 2000. Asia and South Pacific, pp. 191-196, 2000.

[23] W. Krenik, D. D. Buss, and P. Rickert. "Cellular handset integration-SIP versus SOC." IEEE journal of solid-state circuits 40, no. 9, 1839-1846, 2005.

[24] C. H. Douglas. "Wafer level system integration for SiP." In 2014 IEEE International Electron Devices Meeting (IEDM), pp. 27-1, 2014.

[25] R. Tummala. "SOP: what is it and why? A new microsystem-integration technology paradigm-Moore's law for system integration of miniaturized convergent systems of the next decade." IEEE Transactions on Advanced Packaging, vol. 27(2), pp. 241-249, 2004

[26] Y. Chiu, \& H.C. Hong. "Rigid-Flex PCB Technology with Embedded Fluidic Cavities and Its Application in Electromagnetic Energy Harvesters." Micromachines, 9(6), 308, 2018

[27] A.C. Siegel, S.T. Phillips, M. D. Dickey, N. Lu, Z. Suo, \& G.M. Whitesides. "Foldable printed circuit boards on paper substrates." Advanced Functional Materials, 20(1), 28-35, 2010

[28] A. Salahouelhadj, M. Martiny, S. Mercier, L. Bodin, D. Manteigas, \& B. Stephan, "Reliability of thermally stressed rigid-flex printed circuit boards for High Density Interconnect applications." Microelectronics Reliability, 54(1), 204-213, 2014

[29] X. Li, X. Su, \& Y. Liu. "Cooperative robotic soldering of flexible PCBs." In 2017 IEEE/RSJ International Conference on Intelligent Robots and Systems (IROS), (pp. 1651-1656), 2017

[30] P.Y. Chen, C.W. Wu, \& D.M. Kwai. "On-chip testing of blind and opensleeve TSVs for 3D IC before bonding." In 28th VLSI Test Symposium (VTS) (pp. 263-268), 2010

[31] B. Jordan. "Rigid-Flex PCB Design -A guidebook for designers." Altium Inc., La Jolla, CA, 2013

[32] N. El-Atab, W. Babatain, R. Bahabry, R. Alshanbari, R. Shamsuddin and M. Hussain, "Ultraflexible Corrugated Monocrystalline Silicon Solar Cells with High Efficiency (19\%), Improved Thermal Performance, and Reliability Using Low-Cost Laser Patterning", ACS Applied Materials \& Interfaces, 2019. Available: 10.1021/acsami.9b15175.

[33] N. El-Atab, N. Qaiser, R. Bahabry and M. Hussain, "Corrugation Enabled Asymmetrically Ultrastretchable (95\%) Monocrystalline Silicon Solar Cells with High Efficiency (19\%)", Advanced Energy Materials, vol. 9, no. 45, p. 1902883, 2019. Available: 10.1002/aenm.201902883.

[34] A Nayfeh, A Okyay, N El-Atab, F Cimen, S Alkis "Transparent graphene nanoplatelets for charge storage in memory devices" The Electrochemical Society Meeting Abstracts (ECS), pp. 1879-1879, 2014.
[35] X. Li, J. Feng, E. Wang, S. Meng, J. Klimeš, \& A. Michaelides. "Influence of water on the electronic structure of metal-supported graphene: Insights from van der Waals density functional theory," Physical Review B vol. 85, no. 8, p. 085425, 2012.

[36] I. Rahim, M. Shah, M. Iqbal \& A. Khan. "Synthesis, structural, optical, morphological and multi sensing properties of Graphene based thin film devices." Materials Research Express 2018

[37] N. El-Atab, F. Cimen, S. Alkis, A. Okyay and A. Nayfeh, "Enhanced memory effect with embedded graphene nanoplatelets in $\mathrm{ZnO}$ charge trapping layer", Applied Physics Letters, vol. 105, no. 3, p. 033102, 2014. Available: $10.1063 / 1.4891050$

[38] Xi. Li, Ji Feng, E. Wang, S. Meng, J. Klimeš, and A. Michaelides. "Influence of water on the electronic structure of metal-supported graphene: Insights from van der Waals density functional theory." Physical Review B 85(8), 085425, 2012.

[39] N. El-Atab, B. Turgut, A. Okyay, M. Nayfeh and A. Nayfeh, "Enhanced non-volatile memory characteristics with quattro-layer graphene nanoplatelets vs. 2.85-nm Si nanoparticles with asymmetric $\mathrm{Al} 2 \mathrm{O} 3 / \mathrm{HfO} 2$ tunnel oxide", Nanoscale Research Letters, vol. 10, no. 1, 2015. Available: 10.1186/s11671-015-0957-5.

[40] A. D. Smith, Frank Niklaus, A. Paussa, Sam Vaziri, Andreas C. Fischer, Mikael Sterner, Fredrik Forsberg et al. "Electromechanical piezoresistive sensing in suspended graphene membranes." Nano letters, 13(7): 32373242, 2013

[41] B. Mailly-Giacchetti, A. Hsu, H. Wang, V. Vinciguerra, F. Pappalardo, L. Occhipinti, E. Guidetti, S. Coffa, J. Kong, and T. Palacios. "pH sensing properties of graphene solution-gated field-effect transistors." Journal of Applied Physics, 114(8), 084505, 2013.

[42] P. Kailian Ang, W. Chen, A. Thye Shen Wee, and K. Ping Loh. "Solution-gated epitaxial graphene as $\mathrm{pH}$ sensor." Journal of the American Chemical Society, 130(44), 14392-14393, 2008.

[43] H. Cong and T. Pan. "Photopatternable conductive PDMS materials for microfabrication." Advanced Functional Materials, vol. 18(13), pp. 19121921,2008

[44] N. El-Atab and A. Nayfeh, "1D versus 3D quantum confinement in 1-5 $\mathrm{nm} \mathrm{ZnO}$ nanoparticle agglomerations for application in charge-trapping memory devices", Nanotechnology, vol. 27, no. 27, p. 275205, 2016. Available: 10.1088/0957-4484/27/27/275205.

[45] N. El-Atab et al., "Cubic-phase zirconia nano-island growth using atomic layer deposition and application in low-power charge-trapping nonvolatile-memory devices", Nanotechnology, vol. 28, no. 44, p. 445201, 2017. Available: 10.1088/1361-6528/aa87e5.

[46] N. El-Atab et al., " 3-nm ZnO Nanoislands Deposition and Application in Charge Trapping Memory Grown by Single ALD Step", Scientific Reports, vol. 6, no. 1, 2016. Available: 10.1038/srep38712. 